\title{
AutoeVAluACIÓN: PARTICIPACIÓN, CULTURA Y PRINCIPIOS
}

\section{SELF: PARTICIPATION, CULTURE AND PRINCIPLES}

\author{
Francisco Torres Cornejo \\ Licenciado en Administración, \\ Alumno de la Maestría en Educación: Área Gestión \\ Educativa. \\ f_t_cornejo2011@hotmail.com
}

\section{Resumen}

El presente ensayo es una reflexión acerca de la autoevaluación en instituciones educativas, se inicia con algunos antecedentes en la década de 1990. Se analizan conceptos de evaluación y autoevaluación, se hace hincapié en que la finalidad es elaborar juicios que sirvan para tomar decisiones que conduzcan a la autorregulación, es decir, a la mejora.

Se habla de generar una cultura de la evaluación que promueva la participación de los miembros de la comunidad educativa con honestidad y responsabilidad; lo que implica la enunciación de nuestra noción de calidad educativa. Las fases metodológicas de la autoevaluación se deben realizar con el compromiso de mejorar y no sólo para cumplir con la normatividad siguiendo ciertos principios que al final de este trabajo se mencionan.

Palabras clave: Autoevaluación, cultura, participación, calidad, principios. 


\section{Abstract}

This essay is a reflection on the self in educational institutions, beginning with some background in the 1990's. Concepts are analyzed and self-evaluation, emphasizing that the aim is making judgments, which serve to make decisions that lead to self-regulation (improvement).

There is talk of creating a culture of assessment that promotes the participation of members of the educational community with honesty and responsibility, which implies the statement of our notion of educational quality. The methodological phases of self-evaluation should be conducted with a commitment to improve, not only to comply with regulations, according to certain principles that are mentioned at the end.

Keywords: Self-assessment, culture, participation, quality, principles.

\section{Introducción}

La autoevaluación, como parte de un proceso de acreditación en las instituciones educativas, ha tomado fuerza como herramienta para recopilar datos sobre el funcionamiento de las escuelas. La evaluación, en general, es concebida erróneamente por los miembros de la comunidad educativa; y en muchos casos provoca suspicacias. Es entendida por una gran variedad de autores como medio para mejorar los procesos con miras a garantizar la calidad educativa. 
En la autoevaluación, es de suma importancia que los integrantes de la escuela tengan el conocimiento sobre los propósitos, objetos a evaluar y alcance con la finalidad de asegurar su participación sin prejuicios, con responsabilidad y honestidad para lograr el éxito de la misma. Por lo tanto, el objetivo de este artículo es analizar la importancia de la participación, la generación de la cultura de la evaluación, la noción de la calidad educativa y los principios para el proceso de autoevaluación.

\section{Antecedentes}

Comenzaremos por decir que a mediados de 1989, se crea la Comisión Nacional de evaluación (CONAEVA), cuyas principales líneas de investigación son la autoevaluación, la evaluación del sistema y la evaluación a los diversos subsistemas (Rubio, 2007). Desde la década de 1990 la evaluación ha tomado relevancia sobre todo para la educación superior, y se ha utilizado como herramienta para detectar fortalezas y debilidades; con la finalidad de utilizar los resultados para mejorar los procesos educativos (Rueda, 2010).

\section{Definiciones y relevancia de la autoevaluación}

La evaluación educativa se entiende como "el proceso mediante el cual se recoge y se interpreta, formal y sistemáticamente, información sobre un programa de formación, se generan juicios de valor a partir de esa información y se toman decisiones para revisarlo, ajustarlo, mejorarlo, fortalecerlo o consolidarlo" (Abreu, Martos y Cruz 2009 mencionado por Cruz, 2010, p. 2). 
Para Santos Guerra mencionado, por Gairín (1993, p.2):

La evaluación interna es una exigencia de la propia actividad, resulta lamentable que los centros escolares vivan independientemente de su éxito. Es más, sin definir exactamente en qué consiste el éxito, resulta cuando menos ingenuo hacer descansar la valoración de la calidad de un centro en las calificaciones obtenidas por los escolares. Si así fuera, bastaría con que todas fueran excelentes.

La autoevaluación se define, según Martín (1994, mencionado por Salazar y Ureña 2002, p.229) como "un proceso de evaluación donde los esfuerzos se centran en que los componentes asumen el protagonismo de ser ellos mismos sujetos, además de ser objeto de evaluación".

"A nivel institucional significa una revisión crítica, integral y sistemática de las actividades y resultados de prever y reorientar los procesos". (CSUCA 1998, mencionado por Salazar, Ureña 2002, p. 229).

Según Landi y Palacios (2010, p. 175) la autoevaluación "otorga las bases para la formulación de juicios de valor en el proceso de evaluación; juicios que a su vez, permitirán tomar las decisiones posteriores de manera racional, de acuerdo con las finalidades perseguidas por la evaluación".

La autoevaluación es mencionada por el Consejo nacional para la acreditación superior A.C., como una etapa del Proceso de Acreditación dentro del Marco General para los Procesos de Acreditación de Programas Académicos 
de Nivel Superior (COPAES,2012). Sin embargo, esta etapa no es privativa para las Instituciones de Educación Superior (IES), los actores de instituciones de Educación Básica y Educación Media Superior, pueden practicarla optativamente para mejorar sus procesos; independientemente para una acreditación.

"La autoevaluación ha causado interés en la medida que ha sido señalada como recurso para valorar el estado que guardan las instituciones, los programas, los profesores, y los estudiantes" (Rueda 2010). En este sentido, Salazar y Ureña (2002, p. 241) señalan que: "Después de la autoevaluación, viene la autorregulación, sentida con una necesidad no sólo para garantizar la calidad institucional sino además, porque involucraría a todos los que conforman la institución en forma constructiva y reconstructiva para la propia realidad".

Se entiende por autorregulación: el proceso mediante el cual participan los miembros de la comunidad educativa con el objeto de implementar mejoras. Entonces, la institución realiza la autoevaluación con la finalidad de detectar fortalezas y debilidades, las cuales se analizan y se llega a conclusiones que serán el fundamento para la toma de decisiones, que se implementarán en una respuesta de autorregulación.

Castillo, citado por Salazar y Ureña, (2002, p. 233), menciona que a la autorregulación:

Corresponde a la puesta en práctica de los planes de mejoramiento, y las actividades de seguimiento de parte de los propios interesados. Es una mejora continua y 
sistemática que dará lugar en forma natural a cambio, como parte de un proceso permanente.

Podemos decir que la autoevaluación es una evaluación que realizan los miembros de la institución educativa, con la finalidad de detectar deficiencias en programas y/o procesos institucionales, se integran en un informe para analizarlos y se elaboran juicios para tomar decisiones; con la participación de todos se elaboran planes de acción con el objeto de mejorar (autorregulación) en un proceso cíclico.

Lo anterior conlleva a la participación y responsabilidad de los miembros de la comunidad escolar (Directivos, Personal de Apoyo, Docentes, Alumnos y Padres de Familia).

Sólo puede llevarse a cabo la autoevaluación en la medida que exista el compromiso y deseo por elevar la calidad de lo que se ha hecho hasta el momento. En caso contrario puede constituirse en un trámite administrativo más, que lejos de desencadenar un proceso de mejora, sea sólo una simulación, provoque la desatención del trabajo académico (SEP, 2007, p. 9).

Landi, Palacios (2010, p.159), mencionan:

Vale para generar conocimiento más profundo acerca de una determinada realidad. Este conocimiento requiere de la formulación de interpretaciones acerca del valor del quehacer cotidiano de los miembros de la organización. El desafío es promover una reflexión colectiva para producir cambios que impulsen un constante proceso de mejora. 
El proceso no es sólo técnico, también deberá haber acciones éticas y políticas que gestionen la transformación de la función pedagógica de calidad en un contexto de trabajo colaborativo (Landi, Palacios, 2010).

Por tal razón, la autoevaluación no es un proceso fácil y no debe hacerse en cualquier momento, deberá existir el ambiente propicio para la ejecución y que no afecten el desarrollo del mismo. Entonces, cabe preguntarse: ¿Cuáles son las características y condiciones en las que se debe desarrollar una autoevaluación en la institución? Tomaremos las medidas que sugieren Landi y Palacios (2010) para la autoevaluación institucional.

Características de la autoevaluación institucional (Al)

- Los actores de la organización escolar son quienes conducen e implementan el proceso.

- Se pueden procurar asesores o personal externo en el proceso sólo si fuera necesario y existieran dificultades de gestión.

- La finalidad es fortalecer los mecanismos de autorregulación institucionales.

- Las dimensiones, aspectos y criterios utilizados para esta autoevaluación son previamente seleccionados por la propia institución.

- Su producto es un informe de autoevaluación con acciones de mejora a ser implementadas para optimizar la calidad de la formación y finalidades educativas que se persiguen.

Condiciones que posibilitan la autoevaluación institucional (Al) 
- La cultura de la participación. Es necesario comprender cómo construirla.

- La voluntad política para realizar la Al sin la cual ésta fracasa.

- El compromiso y la participación activa de los miembros de la comunidad durante todo el proceso.

- La viabilidad del acceso a la información a ser analizada.

- El apoyo del personal técnico para el procesamiento de la información.

- La utilización de los resultados para proponer los planes de mejora.

Con las características y condiciones que anteriormente se mencionaron, aseguramos el éxito de la autoevaluación. Por lo tanto, el director deberá propiciar una cultura de participación y evaluación, en la que todos aporten y enriquezcan el trabajo colaborativo.

\section{La Cultura de la autoevaluación}

En el marco de una organización escolar, la cultura se refiere a "las creencias y convicciones básicas que mantienen los miembros de la comunidad educativa en relación con la enseñanza, el aprendizaje, la evaluación y en general, con el funcionamiento institucional" (Valenzuela, Ramírez y Alfaro 2011, p. 44).

Una cultura de evaluación podría definirse, entonces, como "el conjunto de valores acuerdos, tradicionales, creencias y pensamientos que una comunidad educativa asignan a la evaluación". (Valenzuela et al., 2011 p.45). 
Podemos comentar que en las instituciones hay una gran variedad de opiniones, comentarios y pensamientos con relación a diversos temas. El término evaluación produce, en la mayoría de las ocasiones, un sentimiento de miedo o culpa.

Los directivos de las escuelas e instituciones deberán promover acciones que eviten expresiones "negativas", reflejo del pensar y sentir de los miembros de la comunidad (Valenzuela et al., 2011). Sensibilizando sobre la importancia de este proceso, haciendo relevante el uso de los resultados para mejorar procesos deficientes y mantener los que están en un nivel aceptable; dilucidando dudas que pueda haber al respecto.

En otras palabras, se deberá de trabajar en propiciar una cultura en el que cambien la forma de pensar de los miembros de la escuela con respecto a la autoevaluación; y en seguida, lograr motivar a docentes y personal de apoyo, para que cooperen, participen y aporten a este proceso.

La participación de directivos, docentes, personal de apoyo y padres de familia está presente en las diferentes etapas de la Autoevaluación, dentro de las fases metodológicas propuestas por Landi y Palacios (2010, pp. 170-175) retomo las que comentaré a continuación:

\section{Fases metodológicas de la autoevaluación}

Identificar el objeto a evaluar.- Conviene preguntar sobre qué evaluar en la institución, respecto a la cual hay diversas dimensiones como: el clima organizacional, 
investigación pedagógica, recursos tecnológicos, prácticas de enseñanza y de evaluación, el análisis de las prácticas educativas y los aprendizajes, el uso de las Tecnologías de la Información y Comunicación (TICS) y el espacio ampliado y contextual de la escuela.

Articular las dimensiones.- La definición del objeto a evaluar, está en función de la finalidad de la misma; e implica el trabajo con varias dimensiones: objeto, referentes, finalidades, comunicación, evaluador, etc. Las finalidades pueden ser la autorregulación, la diagnóstica y la formativa.

Determinar los criterios de evaluación.- Los miembros de la comunidad educativa, deberán hacer una reflexión profunda; sobre los criterios que les permitirán analizar situaciones y discernir sobre los procesos y resultados.

Buscar indicios.- Implica buscar señales de aquellas cosas donde no se puede acceder de manera directa, por lo tanto, la compresión de lo que se obtiene nos permite realizar estimaciones, pero, no brinda evidencias absolutas.

Analizar e interpretar.- Se realizará el análisis y la interpretación de los resultados, sobre la base de los criterios establecidos anteriormente definidos.

Elaborar informes.- En este punto Landi y Palacios, (2010, p. 175) comentan lo siguiente: "Esta etapa del proceso tiene directa relación con uno de los requisitos esenciales de la evaluación con miras a la Al, pues se trata de compartir los resultados obtenidos con muchos más destinatarios e interesados, además del propio 
alumno". Se habla de hacer públicos los resultados y que todos conozcan.

Tomar decisiones.- Es la parte final del proceso de autoevaluación, cuyo objetivo es generar información retroalimentadora que servirá para la toma de decisiones que se deriva de la información recabada.

En cada una de estas etapas están involucrados los integrantes de la comunidad educativa, el personal directivo interviene en todas las fases, $y$ todo el personal deberá participar en la toma de decisiones llegando a acuerdos de manera democrática. Sin embargo, deberá existir una motivación que asegure la participación de todos, desde proporcionar información, la recolección de datos hasta la estructuración de la información y la implementación de acciones. Wells (1998) citado por Salazar, Ureña (2002, p.241), menciona "una motivación desde adentro y no percibida como una obligación, esto nos llevaría a preguntar cuál es el motivo que orienta la realización de estos procesos de acreditación". Lo que quiere decir, es que debemos comprometernos hacia la mejora de nuestra noción de calidad educativa Santos (2009), porque así lo queremos para el bien de nuestra escuela, alumnos y comunidad; la autoevaluación nos ayudará para este propósito y no sólo para cumplir la normatividad de la autoridad educativa.

\section{La autoevaluación para lograr la calidad educativa}

Santos (2009), sugiere que para evaluar la calidad educativa se debe partir de una noción del concepto de calidad. La noción del Instituto Nacional de Evaluación 
Educativa (INEE) asegura que un sistema educativo es de buena calidad si:

- Establece un currículo adecuado a las circunstancias de la vida de los alumnos (Pertinencia) y a las necesidades de la sociedad. (Relevancia)

- Permanezca en ella hasta el final del trayecto y egrese alcanzando los objetivos. (Eficiencia interna)

- La educación será fructífera para la sociedad e individuos. (Eficiencia externa e impacto)

- Recursos humanos y materiales necesarios (Suficiencia), evitando derroches y gastos innecesarios. (Eficiencia)

- Los objetivos educativos sean alcanzados por el mayor número posible de estudiantes. (Equidad)

Los puntos anteriores son ejemplos de criterios de evaluación (la institución puede plantearse otros), que pueden medirse en la autoevaluación y nos indicará cuál es la posición real de la escuela, estos criterios pueden servir de comparativo con otras instituciones y saber el nivel de competencia. Además, saber qué acciones de mejora están implementando otras escuelas y cuáles han resultado exitosas; y si es posible implementarlas en la nuestra.

\section{Principios de la autoevaluación}

Es importante que la autoevaluación que emprende la institución educativa se guíe con ciertos principios que aseguren la honestidad y compromiso de los integrantes de la misma, Correa (2004), propone los siguientes principios para la autoevaluación y aseguramiento de la calidad. 
Institucional: Llevar a la práctica un proceso de autoevaluación como aspecto fundamental de la cultura institucional, requiere que éste tenga un reconocimiento y legitimación institucional, el cual debe ser plenamente reconocido por los diferentes estamentos que lo conforman.

Independiente de la acreditación: No se concibe ni se aplica con el único objetivo de responder a las exigencias de normatividades relacionadas con la acreditación del servicio que se presta. Obedece a la lógica activa y del compromiso de sus miembros.

Ética: En toda organización educativa, debe tener su código ético, centrado en la determinación clara de sus fines misionales, para producir un bien por el cual cobra legitimidad; qué medios utiliza para producir el servicio social de la educación; es desarrollar procesos formativos educativos de calidad, de acuerdo a las necesidades y expectativas de la sociedad y respetar los derechos reconocidos por la sociedad y los valores que se comparten en la misma.

Integral: El proceso de autoevaluación institucional, debe ser sistemático, dialógico, y holístico, para no dejar de lado, ninguno de los procesos y aspectos que lo componen por sencillo que parezcan.

Analítica: La autoevaluación obedece a la lógica de construir nuevas estructuras cognitivas, valorativas, de acción y de gestión, con respecto a su objeto social; de allí el porqué, se desarrolla a la luz de los procesos de análisis y síntesis propuestos de determinadas 
concepciones metodológicas, sean estas participativas, de acción participación, evaluativos, investigativas, entre otras.

Gestionaría: El proceso de autoevaluación debe ser movilizador de voluntades y responsabilidades para posicionar la institución en el contexto, hacerla permeable de cambios. Una gestión fundamentada en la reflexión de las finalidades de la institución y apoyada en la participación real de los estamentos, la cual contribuye a desarrollar la responsabilidad y la capacidad de diálogo, de escucha activa, de planificación, administración, ejecución, seguimiento y evaluación al aprendizaje del trabajo en equipo.

Comparativa: El proceso de autoevaluación centrado en un determinado modelo, proporciona dimensiones, categorías, variables, logros e indicadores que orientan el proceso de recolección de datos, cuyo análisis viabiliza la comparación con los fines, objetivos y metas establecidas en determinados tiempos y espacios.

Fáctica: Todos sus procesos, componentes, acciones y resultados deben ser susceptibles de verificación, mediante la implementación de un determinado diseño; sea este cualitativo, cuantitativo o integral.

Apoyarse en sistemas de información requiere que esté soportada en un sistema de información tanto de la dinámica institucional como del contexto externo, toda vez que la autoevaluación, debe ubicarse en la relación dialógica de estos contextos. Como resultado de este principio, gradualmente se va sistematizando una buena 
base de datos confiables, de gran importancia para la vida de la institución.

Aplicable tanto en su proceso como en sus resultados: La Institución como organización posee la capacidad de aprender, desaprender, y reaprender para emprender; sin embargo, para que este proceso sea realidad, demanda voluntad, disposición, compromiso y dinamización para poder comprender la importancia de los resultados de la evaluación.

\section{Conclusiones}

En las organizaciones de cualquier índole, hay cierta incertidumbre acerca de los procesos de autoevaluación. En mi experiencia laboral he observado que no hay un compromiso sólido para elaborar este proceso con honestidad y responsabilidad, asimismo, darle la importancia requerida. La participación que se llega a dar es por obligación para cumplir la normatividad impuesta por las autoridades educativas, o para cumplir procesos de acreditación. En la mayoría de los casos no se ve como herramienta de reflexión para mejorar los procesos educativos.

En los subsistemas educativos se observa un área de evaluación sin realizar la función como tal; se limita sólo al seguimiento de los programas y a la aplicación de los recursos financieros, dejando a un lado los procesos inherentes a la educación; debiendo estar supeditada su función a decisiones de intereses particulares, sobre todo políticos. 
Los departamentos de evaluación, cuyo nombre real bien podría ser departamentos de autoevaluación, (porque ésta es realizada por personal perteneciente a la institución) deberían actuar como elementos de apoyo elaborando metodologías para la evaluación de procesos de trabajo, programas y desempeño; con la finalidad de proporcionar información relevante para la toma de decisiones en pro de la autorregulación (mejora), de las actividades académicas.

En instituciones escolares especialmente en las descentralizadas se realizan una evaluación al personal, no con el ánimo de mejorar, sino, porque de acuerdo a la LFPRH ART. 45 (2008), la asignación del presupuesto estará determinada por los resultados que se generen. Lo que causa suspicacias al personal, sobre todo en un ambiente que no se ha preparado para este proceso. Se tiene el error de evaluar para cumplir la normatividad, y la evaluación no es vista como una herramienta para llevar a cabo una reflexión de nuestra labor educativa, y por lo tanto contribuir a nuestra noción de calidad (Santos, 2009).

Desde el punto de vista de la equidad, no es viable otorgar recursos con base en resultados. Porque no todos los subsistemas de los Estados de la República tienen las mismas oportunidades de desarrollo.

Generar un ambiente y gestionar una cultura de la participación para la autoevaluación, es responsabilidad de los directivos. Se debe acabar con "fantasmas" reconociendo el trabajo de quienes participan en ella, sensibilizando al personal sobre la importancia y 
responsabilidad de su aportación, así como, la contribución de todos los integrantes hacia la mejora de la calidad educativa; lo anterior es responsabilidad de quienes dirigen las escuelas.

La participación de los miembros de la institución educativa es primordial para el éxito de la autoevaluación. Generar una cultura de la evaluación traerá como beneficio el trabajo colaborativo enmarcado en un proceso constante y cotidiano e inmerso en principios y valores organizacionales.

\section{Fuentes de CONSULTA}

- COPAES Consejo para la Acreditación de la Educación Superior, A.C. (2012). Marco General para los Procesos de Acreditación de Programas Académicos de Nivel Superior. México.

- CORREA DE MOLINA, Cecilia (2004). Gestión y Evaluación de la calidad en la educación: Referentes generales para la acreditación. Colombia: Cooperativa Editorial Magisterio.

- CRUZ CARDONA, Víctor (2010). Evaluación de la calidad de los programas de maestría y doctorado en Iberoamérica. Revista Digital Universitaria, 11, 5, 3-10.

- GAIRÍN SALLÁN, Joaquín (1993). La autoevaluación institucional como vía para mejorar los centros educativos. [versión electrónica]. Bordón Revista de Pedagogía, 45, 3, 331-350. 
- LANDI, Nidia Edith y Palacios, María Elena (2010). La autoevaluación institucional y la cultura de la participación. [versión electrónica]. Revista Iberoamericana de Educación, 53, 155-181.

- MÉXICO. Cámara de Diputados del H. Congreso de la Unión (2008). Ley Federal de Presupuesto y Responsabilidad Hacendaria: LFPRH.

- RUBIO OCA, Julio (2007). La evaluación y acreditación de la educación superior en México: un largo camino aún por recorrer. [versión electrónica]. Reencuentro, 50, 35-44.

- RUEDA BELTRÁN, Mario (2010). Autoevaluación y autonomía. [versión electrónica]. Perfiles Educativos, XXXII, 130, 3-6

- SALAZAR FRANCIS, Susan y Ureña Salazar, Viria. (2002). El proceso de autoevaluación y autorregulación de la sede del Atlántico de la Universidad de Costa Rica: una mirada a la opinión de sus participantes tres años después. [versión electrónica]. Revista de la Universidad de Costa Rica, 26, 002, 229-243.

- SANTOS DEL REAL, Annette (2009). La evaluación como herramienta de mejora de la calidad educativa. Revista CNEP, 33, 13-15.

- SEP Secretaria de Educación Pública. (2003). ¿Qué tan buena es nuestra Escuela? México. 
- SEP Secretaria de Educación Pública. (2007). ¿Cómo puedo Mejorar Mi Escuela? México.

- VAlenZUELA GONZÁleZ, Jaime Ricardo, Ramírez Montoya, María Soledad y Alfaro Rivera, Jorge Antonio (2011). Cultura de evaluación en instituciones educativas, comprensión de indicadores, competencias y valores subyacentes. [versión electrónica]. Perfiles Educativos, XXXIII, 131, 42-63. 
